Address for Correspondence: Prof. Bei Wang,

Department of Respiration, Second Hospital of Shanxi Medical University, Taiyuan 030001, China.

E-mail: myemaily@sohu.com

Chief Physician, Master tutor, Director

of Diagnosis/Therapy Center for

Sleep Breath Disorder, Department

of Respiration, Second

Shanxi Medical University. She is

also a member of Sleep Study Group of Respiratory Diseases Society of Chinese Medical Association, and an expert commissioner of Chinese Sleep Research Society. She has published more than 50 papers in foreign and

domestic key journals.

\begin{tabular}{|l|}
\hline Access this article online \\
\hline $\begin{array}{l}\text { Website: } \\
\text { www.intern-med.com }\end{array}$ \\
\hline DOI: \\
10.1515/jtim-2013-0004 \\
\hline Quick Response Code: \\
\hline \\
\\
\hline
\end{tabular}

\title{
Obstructive sleep apnea syndrome and dilated cardiomyopathy
}

\author{
Bei Wang
}

Department of Respiration, Second Hospital of Shanxi Medical University, Taiyuan 030001, Shanxi Province, China

\section{ABSTRACT}

With in-depth understanding of obstructive sleep apnea syndrome (OSAS), the correlation between OSAS and various cardiovascular diseases receives increasing attention. Together with Chinese Society of Cardiology, Sleep Breath Disorder Group of Chinese Society of Respiratory Diseases issued an "expert consensus on sleep apnea and cardiovascular disease" in light of the relevant foreign documents in 2009 , which became the new starting point to further promote and closely connected the two interdisciplinary researches. This paper gives a brief introduction of how to early recognize and diagnose OSAS complicated with concurrent dilated cardiomyopathy (DCM), emphasizes the clinical significance of diagnosis in such patients, and analyzes the clinical value of continuous positive airway pressure treatment in restoring the cardiac function and structure in DCM patients.

Key words: dilated cardiomyopathy, obstructive, sleep apnea

\section{A CASE INTRODUCTION}

A 59-year-old male patient had recurrent night dyspnea and edema in both lower limbs for 10 years. He was seen in the office with a chief complaint of aggravated symptoms accompanied with bradycardia. In his local hospital, he was diagnosed as dilated cardiomyopathy (DCM) with atrioventricular conduction block Grade III through the heart color ultrasonic examination and $24 \mathrm{~h}$ dynamic electrocardiogram (ECG) examination. After implantation of the two-chamber cardiac pacemaker and administration of routine medicines in that hospital (i.e., oral Enalapril (an angiotensin converting enzyme inhibitor [ACEI], $25 \mathrm{mg}$, q.d; oral Hydrochlorothiazide [25 mg, b.i.d]; and intravenously-dripped drugs for improving myocardial nutrition and energy metabolism), however, the symptoms were not alleviated significantly and recurrent bradycardia and night dyspnea still occurred. After replacement with three-chamber cardiac pacemaker in that hospital, the symptoms still was not been improved, furthermore, coma developed gradually in the patient. Then, he was referred to ICU of our Department of Cardiology. After admission, through relevant cardiac examinations, he was diagnosed again as DCM with GradeIV cardiac insufficiency and Grade-III atrioventricular conduction block. After intravenous dripping of Aminophylline (0.25 g, b.i.d) and continuous pumping (0.8-1.5 mL/h) of Sodium Nitroprusside $(50 \mathrm{mg})$ and Normal Saline $(50 \mathrm{~mL})$, both atrioventricular conduction block and night dyspnea were still not alleviated significantly. During the joint consultation by Department of Respiration, he complained of 20-year snoring and 10-year significant weight gain with concurrent daytime sleepiness. Finally, he was diagnosed as severe obstructive sleep apnea syndrome (OSAS) through polysomnography (PSG) with sleep apnea hyponea index (AHI) 58 (mainly of obstructive type), maximum apnea duration $112 \mathrm{~s}$ and minimum night oxygen saturation $58 \%$. As examined by the Ear-Nose-Throat Department, the oral, nasal and pharyngeal cavities were not significantly obstructed. He was treated with BiPAP non-invasive ventilator. Night dyspnea disappeared $3 \mathrm{~d}$ later; Grade III atrioventricular conduction block improved significantly 2 weeks later, 
and disappeared $20 \mathrm{~d}$ later; the cardiac color ultrasonic examination and ECG were performed again 10 weeks, 6 months and 26 months later, respectively and found no Grade III atrioventricular conduction block, narrowed atria and ventricles in various degrees, normal cardiac function, and no edema in both lower limbs). Then, the cardiac pacemaker was removed and the symptoms did not appear any more.

This is a typical case of OSAS with concurrent DCM. Among the sleep dyspnea diseases, OSAS has the highest incidence and is most harmful to human health and life. In OSAS (with an incidence rate of about $4 \%$ in adults), the upper airway is completely or incompletely obstructed repeatedly during the sleep, causing frequent apnea or hyponea. ${ }^{[1]}$ As uniformly verified from different aspects by epidemiological surveys, clinical trials and experimental basic researches, OSAS highly relates to various cardiovascular diseases, and significantly increases the incidence rate and death rate of cardiovascular diseases (e.g., hypertension, coronary heart diseases, arrhythmia, pulmonary artery hypertension and heart failure). ${ }^{[2]} \mathrm{At}$ present, OSAS has become an important risk factor for the occurrence and development of various cardiovascular diseases and the untreated OSAS has also become an independent risk factor for increasing the death rate of cardiovascular diseases. ${ }^{[3]}$

DCM is a myocardial disease of unknown cause with ventricular enlargement and myocardial systolic dysfunction. Besides coronary heart diseases and hypertension, DCM mainly causes heart failure. In recent years, the incidence rate of DCM tends to increase gradually in China. As shown in current researches, DCM is caused by the abnormal controlling gene of myocardial cells, viral infection, immune dysfunction and mechanical pressure..$^{[4]}$ Such factors gradually lead to degeneration, necrosis, apoptosis, fibration and interstitial edema of myocardial cells; the heart is remodeled (e.g., chamber enlargement and ventricular wall thinning) and the cardiac function weakens gradually, causing heart failure. In clinical practice, DCM is mainly treated with the following measures: Relieving cardiac pre-/after-load, nourishing the myocardium, improving the metabolism, increasing myocardial contractility and correcting cardiac function.

In clinical practice, some DCM patients have the concurrent OSAS in various degrees. After treatment with non-invasive ventilator, their cardiac function improves significantly, and some cardiac structures change reversibly. Therefore, it is worth exploring the correlation between OSAS and DCM. Above all, we must be clear under what conditions DCM with concurrent OSAS is considered and whether the therapeutic outcome of DCM will be influenced seriously by the existence of OSAS. If there is still frequent refractory heart failure (or slow arrhythmia, e.g., bradycardia) during night sleep after the routine standard therapy (e.g., cardiotonic, diuresis, vasodilatation, combined ACEI, calcium channel blocker and $\beta$ receptor blocker), DCM patients are highly likely to have OSAS. Then, with the existing obesity and lip cyanosis or other symptoms in the past (e.g., snoring at night sleep, dry mouth in the morning, headache, daytime sleepiness and hypomnesia), DCM patients should undergo PSG for definite OSAS diagnosis. At present, there are yet no large-scale multicenter clinical trials on verifying the correlation between OSAS and DCM. Some findings of current researches will be introduced hereinafter.

\section{OSAS MECHANISM OF CAUSING CARDIOVASCULAR DISEASES}

The main pathophysiological changes of OSAS include chronic intermittent hypoxia, carbon dioxide retention, thoracic negative pressure increase, recurrent movement arousal and abnormal sleep structure. On this basis, through a series of changes, these pathophysiological changes lead to violent fluctuation of blood pressure and heart rate, and disfunction of cardiac automaticity. Current studies find OSAS may cause cardiovascular diseases through the following mechanisms. ${ }^{[5]}$

\section{Oxidation stress and inflammatory reactions}

Atherosclerosis and thrombosis are the pathophysiological basis of cardiovascular diseases. Atherosclerosis is a process of chronic inflammation and so is OSAS as shown by recent researches. OSAS can mainly increase the following four plasma inflammatory cell factors: (1) Intercellular adhesion molecule-1, vascular-cellular adhesion molecule-1 and P-selectin; (2) inflammatory medium and cell factors (e.g., IL-1, IL-6 and TNF- $\alpha$ ); (3) C-reactive protein; and (4) matrix metalloproteinases. These cell factors cooperatively promote the formation and development of atheromatous plaque.

\section{Vascular endothelial dysfunction}

Endothelial cells maintain the intact function of blood vessel by synthesizing and releasing various bio-active substances and keep the balance between vasoconstriction and vasodilatation by regulating the vascular response to adapt to different physical or chemical stimulus, whose unbalance can damage the endothelial function. In OSAS patients, due to the chronic intermittent hypoxia and re-oxygenation, both the induced oxidation stress and inflammatory reactions can cause the endothelial dysfunction and accelerate the occurrence of atherosclerosis and acute coronary syndrome. 
Besides above two main factors, the following factors can cause the onset and progression of cardiovascular diseases in OSAS patients, i.e., autonomic nerve dysfunction, blood viscosity increase, hypercoagulative state, fibrinolytic dysfunction, and endocrine dysbolism.

\section{OSAS IMPACT ON CARDIAC STRUCTURE/FUNCTION}

As verified by foreign and domestic researches, many factors may participate in the process of cardiac remodeling during the pathophysiological occurrence and development of OSAS and greatly affect the cardiac structure/function, i.e., hypertrophy/dilatation in the right ventricle, inner diameter increase in the right pulmonary artery, E/A ratio decrease in the right ventricle, thickness increase in the septum and posterior wall of the left ventricle, output decrease in the left ventricle. ${ }^{[6,7]}$ Its mechanism is shown as follows: (1) The continuous excitability improvement of sympathetic nervous system can increase the blood pressure, accelerate heart rate, increase myocardial oxygen consumption, increase the body concentration of catecholamine substances and down-regulate the density of myocardial $\beta 1$ receptor, so as to cause myocardial remodeling, affect myocardial metabolism, and change normal hemodynamics.(2) The intrathoracic negative pressure can improve the gradient of osmotic pressure through chamber wall and affect the ventricular function, cardiac automaticity and hemodynamic stability, so as to possibly thicken the ventricular wall, increase cardiac pre-/post-load, enlarge the auricle and cause ventricular dyssystole.(3) The recurrent hypoxia and re-oxygenation during the sleep can produce the pathological process like ischemiareperfusion injury to produce numerous free radicals in leukocytes for directly damaging endothelial cells and selectively activate inflammation-promoting transcription factors (e.g., nuclear factor- $x \mathrm{~B}$ ) to release inflammatory factors.(4) OSAS is the risk marker for thrombosis (i.e., the increase of blood viscosity, blood platelet activity and fibrinogen), affects the hemodynamic and causes vascular endothelial dysfunction. As verified by the animal experiments the frequent hypoxia in various degrees first causes multiple small-foci infarction and ischemia of rat myocardium and then causes ventricular remodeling and cardiac hypofunction.

\section{CORRELATIONS BETWEEN OSAS AND DCM}

In the research by Noda et al., ${ }^{[8]} 21$ cases of 52 DCM patients (AHI $\geq 21,40.4 \%$ ) were randomly divided into two groups: Treatment Group (10 cases, routine therapy combined with BiPAP ventilator therapy) and Control Group (11 cases, no BiPAP ventilator therapy). Compared with that in the Control Group, after 3-month therapy, the cardiac function improved significantly and the inner diameter of the left ventricle at the end-systole/diastole decreased significantly in Treatment Group, indicating that non-invasive ventilator therapy not only improved the cardiac function but also narrowed the enlarged left ventricle. Moreover, during the follow-up, there were no deaths in Treatment Group, but 4 deaths (36.4\%) in Control Group. In the research of Wang et al. on 4 DCM patients with concurrent OSAS, ${ }^{[0]}$ after the single routine medicine therapy, the efficacy was not satisfactory with frequent night heart failure and one patient with refractory sinus bradycardia did not yet restore to normal state through the implantation of three-chamber cardiac pacemaker; after 6-28 months of combined therapy with continuous positive airway pressure, OSAS symptoms were alleviated and the cardiac function improved significantly in all subjects whose symptoms were alleviated with ejection fraction increase in left ventricle, inner diameter decrease at the end-diastole in left ventricle and improvement of cardiac function grading, indicating that the cardiac function improved and the partially-enlarged cardiac chamber changed reversibly in the inner diameter.

As verified by the above researches, OSAS and DCM are likely to coexist. At their coexistence, DCM treatment has poor efficacy and prognosis. Therefore, OSAS should be treated (e.g., with non-invasive ventilator) at the same time of DCM pharmacotherapy, so as not only to achieve good efficacy but also to reversibly change some cardiac structures. For DCM patients with concurrent OSAS, the early diagnosis and treatment of OSAS may improve the clinical symptoms, shorten the hospitalization time and improve the quality of life. However, the above two researches only used a small sample, so their conclusion is not very reliable. The correlation between DCM and OSAS should be still verified through the large-sample, randomized and multi-center researches.

\section{IMPACT OF NON-INVASIVE VENTILATOR THERAPY ON CARDIAC FUNCTION/STRUCTURE}

As verified by numerous current researches, non-invasive ventilator therapy can significantly improve cardiac function and plays an irreplaceable role in treating heart failure (especially more significantly in congestive heart failure). It can block the recurrent chronic intermittent hypoxia, re-oxygenation and frequent movement arousal and furthermore antagonize the violent fluctuation of intrathoracic pressure caused by forced inspiration at 
upper air passage blockage, so as to reduce the secretion of adrenaline, lower the excitability of sympathetic nervous system and stabilize the hemodynamics, which can relieve ventricular pre-/post-load, alleviate/diminish the oxidation stress and inflammatory reactions, improve myocardial metabolism and thus improve cardiac function. As also verified by some researches, it can effectively improve cardiac function, weaken several risk factors' impact on cardiac remodeling, ${ }^{[10]}$ effectively prevent the abnormal change in cardiac structure of OSAS patients and reverse their existing structural change to a certain extent. ${ }^{[1]]}$

In a word, the correlation between OSAS and DCM has been verified through literature reviews and researches. The scientific diagnosis and therapy of OSAS is very important and irreplaceable for DCM patients, for it can lower the incidence rate of heart failure, prevent the aggravation of heart failure, overall reduce the disability/death rate, improve the quality of life and prolong the survival time.

\section{REFERENCES}

1. Cardiology Society in Chinese Medical Association and Sleep Breath Disorder Group of Respiratory Diseases Society in Chinese Medical Association, expert consensus on sleep apnea and cardiovascular diseases. Chin J Intern Med 2009;48:1059-67.

2. Budhiraja R, Budhiraja P, Quan SF. Sleep-disordered breathing and cardiovascular disorders. Respir Care 2010;55:1322-32.
3. Marin JM, Carrizo SJ, Vicente E, Agusti AG. Long-term cardiovascular outcomes in men with obstructive sleep apnoea-hypopnoea with or without treatment with continuous positive airway pressure: An observational study. Lancet 2005;365:1046-53.

4. Wang YT, Wang TH. Pathogenesis and therapeutic progress of dilated cardiomyopathy. Chin J Cardiovasc Med 2005;10:230-1.

5. Bradley TD, Floras JS. Obstructive sleep apnoea and its cardiovascular consequences. Lancet 2009;373:82-93.

6. Otto ME, Belohlavek M, Romero-Corral A, Gami AS, Gilman G, Svatikova A, et al. Comparison of cardiac structural and functional changes in obese otherwise healthy adults with versus without obstructive sleep apnea. Am J Cardiol 2007;99:1298-302.

7. Wang B, Liu H, Liu ZL. Impact of obstructive sleep apnea hyponea syndrome on the structure and function of left heart. Chin J Tuberc Res Dis 2007;30:55-6.

8. Noda A, Izawa H, Asano H, Nakata S, Hirashiki A, Murase Y, et al. Beneficial effect of bilevel positive airway pressure on left ventricular function in ambulatory patients with idiopathic dilated cardiomyopathy and central sleep apnea-hypopnea: A preliminary study. Chest 2007;131:1694-701.

9. Wang DP, Wang B, Wang Q, Liu ZL. Clinical experience summary and analysis of continuous positive airway pressure for the obstructive sleep apnea syndrome with concurrent dilated cardiomyopathy. Chin J Tuberc Respir Dis 2010;33:700-2.

10. Kohler M, Pepperell JC, Casadei B, Craig S, Crosthwaite N, Stradling $\mathrm{JR}$, et al. CPAP and measures of cardiovascular risk in males with OSAS. Eur Respir J 2008;32:1488-96.

11. Drager LF, Bortolotto LA, Figueiredo AC, Silva BC, Krieger EM, Lorenzi-Filho G. Obstructive sleep apnea, hypertension, and their interaction on arterial stiffness and heart remodeling. Chest 2007;131:1379-86.

How to cite this article: Wang B. Obstructive sleep apnea syndrome and dilated cardiomyopathy. J Transl Intern Med 2013; 1(1): 9-12.

Source of Support: Nil, Conflict of Interest: None declared 